This paper is a post-print of a paper submitted to and accepted for publication in IET Renewable Power Generation and is subject to Institution of Engineering and Technology. To cite the paper please use the doi : $\underline{10.1049 / i e t-}$ rpg.2018.5728 provided on the IET Digital Library page.

\title{
Comparison of advanced non-parametric models for Wind Turbine Power Curves
}

\author{
Ravi Kumar Pandit ${ }^{1}$, David Infield ${ }^{1}$, Athanasios Kolios ${ }^{2}$ \\ Electronics and Electrical Engineering ${ }^{1}$ and Naval Architecture, Ocean \& Marine Engineering ${ }^{2}$ \\ University of Strathclyde, 16 Richmond St, Glasgow - G1 1XQ, Scotland, UK. \\ Corresponding author Email: ravi.pandit@strath.ac.uk
}

\begin{abstract}
To assess the continuous performance of a wind turbine, accurate power curve modeling is essential. Various statistical methods have been used to fit power curves to performance measurements; these are broadly classified into parametric and nonparametric methods.

In this paper, three advanced nonparametric approaches, namely: Gaussian Process; Random Forest; and Support vector machine are assessed for wind turbine power curve modeling. The modeled power curves are constructed using historical wind turbine SCADA data obtained from operational three bladed pitch regulated wind turbines. The modeled power curve fitting performance is compared using suitable performance error metrics so as to identify the most accurate approach. It is found that a power curve based on a Gaussian Process has the highest fitting accuracy, whereas the Support vector machine approach gives sufficiently accurate results, but within a restricted wind speed range. Power curves based on a Gaussian Process or Support vector machine provide smooth and continuous curves, whereas power curves based on the Random forest technique are neither smooth nor continuous. This paper highlights the strengths and weaknesses of the proposed nonparametric techniques for the purpose of constructing a robust fault detection algorithm for wind turbines based on power curves.
\end{abstract}

\section{Nomenclature}

$\begin{array}{ll}\text { CIs } & \text { - Confidence intervals } \\ \text { COE } & \text { - Cost Of Energy } \\ \text { RF } & \text { - Random Forest } \\ \text { GP } & \text { - Gaussian Process } \\ \text { SVM } & \text { - Support Vector Machine } \\ \text { SVR } & \text { - Support Vector Regression } \\ \text { SVC } & \text { - Support Vector Classification } \\ \text { K } & \text { - The general covariance matrix } \\ \mathrm{k}_{\mathrm{SE}} & \text { - Squared exponential covariance function } \\ \text { MAE } & \text { - Mean absolute error } \\ \text { MSE } & \text { - Mean square error } \\ \text { MAPE } & \text { - Mean absolute percentage error } \\ \text { RMSE } & \text { - Root Mean Square Error } \\ R^{2} & - \text { Coefficient of determination } \\ \text { SCADA } & - \text { Supervisory control and data acquisition } \\ \text { WTs } & - \text { Wind Turbines }\end{array}$

\section{Introduction}

Unexpected failures of wind turbine (WT) components, in particular, the rotor, gearbox, and generator, make operation and maintenance (O\&M) more expensive and can add significantly to the overall Cost Of Energy (COE). Furthermore, offshore wind farm's O\&M cost is higher due to transportation and logistics issues, and thus there is a pressing need to reduce the O\&M cost by employing continuous condition monitoring and using predictive, and proactive maintenance strategies. Predictive maintenance can be useful in identifying failures at an early stage and preventing catastrophic damage. Bently and Hatch [1], define condition monitoring as the process of monitoring the condition of a machine through measurement of parameters

such as vibration or temperature in such way that a significant change is indicative of a developing failure. Such methods are well described in [2-3].

Supervisory control and data acquisition (SCADA) based condition monitoring is considered to be financially savvy since the required information is accessible at no additional cost, see [4,5]. Reflecting this advantage, a number of SCADA based condition monitoring approaches have recently been proposed: vibration for rotor blades [6,7]; advanced signal processing for gearboxes $[8,9]$ or bearings [10]; and for others, see [5,11]. Among the various proposed SCADA based models, the wind turbine power curve is widely used since it reflects the turbine behavior which is helpful for fault diagnosis and condition monitoring. 
This paper is a post-print of a paper submitted to and accepted for publication in IET Renewable Power Generation and is subject to Institution of Engineering and Technology. To cite the paper please use the doi : 10.1049 /iet-rpg.2018.5728 provided on the IET Digital Library page.

Power production is the key consideration when assessing a potential site for wind farm development. The power output of a wind turbine is estimated from the power curve and wind speed profile for the site in question, and also from the site air density as this affects the wind power generation. The predicted long-term gross annual mean power output at a target site is calculated with the help of a WT power curve. Moreover, power curve models can be useful in forecasting and capacity factor estimation purposes. Numerous techniques have been introduced in the past to model WT power curves and these techniques generally divided into parametric and nonparametric. Parametric models are generally based on mathematical models that are often constructed from a family of functions with a number of variables that are fitted to correspond to the particular wind turbine. Widely used parametric approaches are segmented linear models [12], polynomial regression [13], [14], and models based on probabilistic distributions such as four- or five parameter logistic distributions [15], [16]. In contrast to the parametric approach, nonparametric approaches do not enforce any pre-specified condition, and thus, the estimated power curve is as close as possible to the measured data subject to the smoothness of the fit. Because of this, nonparametric models are able to model power curve accurately over a wide range. Several studies have been conducted to develop an accurate power curve for performance evaluation and these methods include a copula power curve model [17], cubic spline interpolation [18], support vector machine [20], neural networks [21,22], and data-driven methods (e.g., Gaussian Process [19], Random Forest [23], and the k-nearest neighbor clustering [24]). A comprehensive review of the existing WT power curve monitoring techniques can be found in [16].

Gaussian Process models are used extensively in the literature $[25,26]$ for a wide range of modelling applications; however, they have not much been used to explored issues related to wind turbines. Support vector machine (SVM) is another nonparametric method that has been introduced for wind turbine power curve modeling [27,28]. However, both methods suffer from a number of practical issues such the cubic inversion issue associated with larger data sets. Finally, the RF model is another nonparametric approach used to construct power curves. Unlike most of the nonparametric approaches, the RF does not need to be tuned or optimized, and it incorporates the prediction of several weak predictors [29]. As the name suggests, it is used to create a forest in some way and make it random while maintaining the direct relationship among the number of trees in the forest. Usually, a large number of trees indicates a more accurate result. It is worth noting that RF and decision tree techniques are not the same because with a RF random samples are used to obtain the root node and splitting the feature nodes, in contrast to decision trees [30]. These advanced nonparametric models are flexible and easy to implement, and computationally straightforward to implement.

\section{Scientific contribution}

As has already been mentioned, power curve model accuracy varies with the techniques and the particular data set used, and hence there is no one technique that performs best in all cases of observations obtained from different wind turbines. It is therefore essential to investigate the performance of different nonparametric techniques for power curve modeling to evaluate which technique is more accurate for a given dataset. Advanced nonparametric models such as the GP, SVM and $\mathrm{RF}$ are gaining popularity because of their low computational cost and high accuracy. The direct comparison between these models can be useful in identifying the method that is more robust and computationally feasible. This paper aims to fill this gap.

The paper presents the implementation of three advanced nonparametric algorithms (GP, SVM, and RF) for modeling of wind turbine power curves, and their accuracy has been compared using error performance metrics (RMSE, $R^{2}$, MAE). Comparison of the methods is made to identify the best approach taking into account the computational cost and required processing power. The SCADA dataset obtained from modern pitch regulated wind turbines is used to train and validate the performance of the proposed nonparametric models. The outcomes should be useful in constructing power curve based fault detection algorithms, where accuracy is paramount, for the purpose of condition monitoring. A framework for modeling wind turbine power curves and its performance comparisons is presented in Figure 1 and described as follows. The SCADA data extracted from operational wind turbines which is then filtered and air density corrected. After this, datasets are divided into training and validation; training data points are used to train the models and validation data points are used to validate the performance of models. Performance Error metrics, residuals analysis and uncertainty analysis, are used to compare the performance of the models and based on this comparison, best approach for Wind Turbine power curve modeling is being suggested.

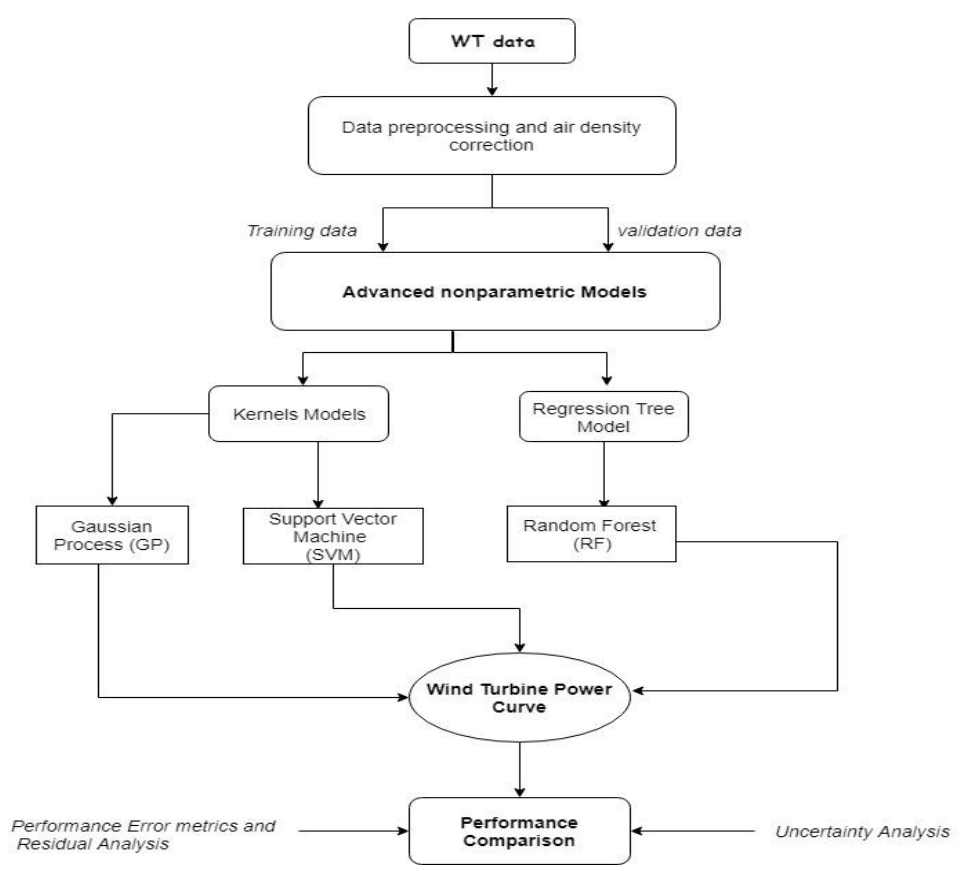

Figure 1: A framework of the advanced nonparametric Wind Turbine Power Curve models for performance comparison 
This paper is a post-print of a paper submitted to and accepted for publication in IET Renewable Power Generation and is subject to Institution of Engineering and Technology. To cite the paper please use the doi : $\underline{10.1049 / i e t-r p g .2018 .5728}$ provided on the IET Digital Library page.

\section{Wind Turbine Power Curve Modeling}

The wind turbine power curve describes the nonlinear relationship between the WT power and hub height wind speed and is shown in Figure 2. Primarily it is used to capture the wind turbine performance. The electrical power output of the turbine is not only correlated with wind speed but also responds to turbulence intensity, wind direction, vertical and horizontal shear, atmospheric stability, drive train temperature, yaw error and so on [31]. An accurate power curve is not only used to improve performance assessments but can play a significant role in identifying different wind turbine fault types. Usually, an individual WT has unique power curve depending to the operating conditions for which it has been designed (e.g., wind speed range) and actively used for continuous monitoring the performance of a wind turbine by differentiating between a normal and an abnormal state [31]. The WT power curve follows the sigmoid shape and any changes in its characteristic shape likely to indicate abnormal operation due to a fault. The wind speed between the cut-in and the cut-out speed ranges are considered significant because this operational region presents a significant opportunity to optimize the wind turbine power generation process. The theoretical power obtained from a wind turbine is given by,

$$
P=0.5 \rho \mathrm{AC}_{\mathrm{p}}(\lambda, \beta) \mathrm{v}^{3}
$$

where $\rho$ is air density $\left(\mathrm{kg} / \mathrm{m}^{3}\right)$, A is swept area $\left(\mathrm{m}^{2}\right), C_{p}$ is the power coefficient of the wind turbine and $v$ is the hub height wind speed $(\mathrm{m} / \mathrm{sec})$. The power coefficient is a function of tip speed ratio $(\lambda)$ and pitch angle $(\beta)$. In addition to these two parameters, the power output of a WT affected by flow conditions, in particular, terrain, wind shear, turbulence intensity and air density [31].

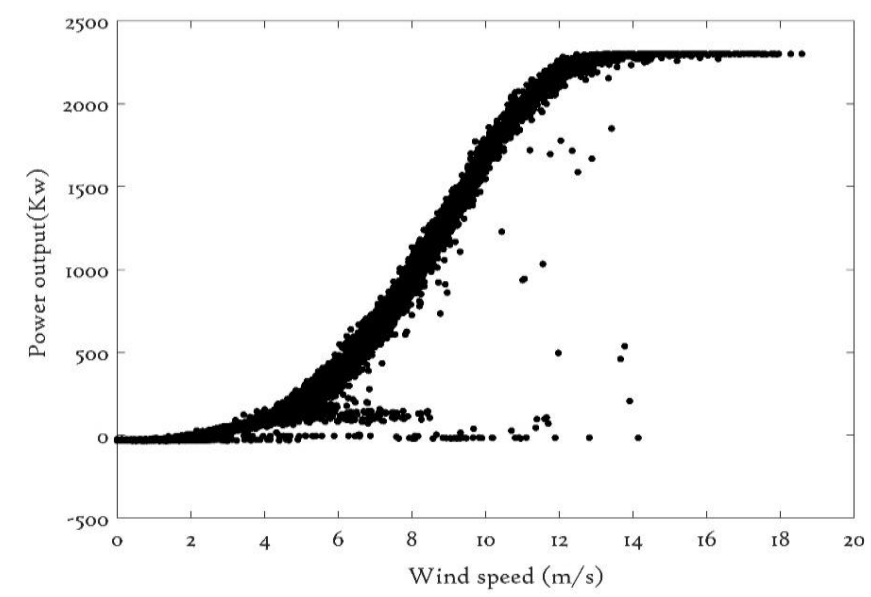

Figure 2: Measured power curve of an industrial wind turbine

\section{Air Density Correction}

The IEC standard (61400-12-1) [32], recommend air density correction for accurate power curve measurement. The IEC standards suggest two approaches for air density correction based on whether the turbine is a pitch or stall regulated. In this paper, SCADA datasets are from a pitchregulated and hence corrected wind speed $V_{C}$ is calculated using equations (2) and (3) as shown below,

$$
\begin{aligned}
& \rho=1.225\left[\frac{288.15}{\mathrm{~T}}\right]\left[\frac{\mathrm{B}}{1013.3}\right] \\
& \text { and, } \mathrm{V}_{\mathrm{C}}=\mathrm{V}_{\mathrm{M}}\left[\frac{\rho}{1.225}\right]^{\frac{1}{3}}
\end{aligned}
$$

where $V_{C}$ and $V_{M}$ are the corrected and measured wind speed in $\mathrm{m} / \mathrm{sec}$ and the corrected air density is calculated by equation (7) where $\mathrm{B}$ is atmospheric pressure in mbar, and $\mathrm{T}$ the temperature in Kelvin in which 10-minute average values obtained from SCADA data are used. The corrected wind speed $\left(V_{C}\right)$ from equation (3) is then used to calculate the power curve, normally by binning. The air density $\rho$ is related to temperature by the gas law $\rho=\frac{p}{(\text { R.T })}$; where $p$ is absolute atmospheric pressure, $\mathrm{R}$ the gas constant and $\mathrm{T}$ is the environmental temperature in Kelvin. The air density computed by using the gas law where air pressure and temperature measured from sonic anemometer at hub height. It is worth to note that because of the significant impact of temperature on air density, air density correction is sometimes referred to as temperature correction.

\section{SCADA data source and pre-processing}

Original Supervisory Control and Data Acquisition (SCADA) based condition monitoring is a cost-effective approach which gathers the information provided by sensors without any extra cost. The wind turbine SCADA system has more than 120 parameters such as the power output, hub height wind speed, ambient pressure, wind direction, vibrations, digital control signals and ambient temperatures; comes with minimum, maximum, average and standard deviation values. These SCADA dataset essential in identifying early warning of failures and improving the performance of WTs. Despite such advantageous; unexpected sensor malfunction makes SCADA datasets erroneous and if such error allowed then affect the model accuracy. Therefore, it is necessary to filter the SCADA data in order to minimize these errors before doing further analysis. The steps described in [33] for example; timestamp mismatches, negative power values, out of range values, abnormal wind speed, and turbine power curtailment considered to remove confusing data. However, it should be noted that despite these adopted methodologies, SCADA data is not entirely free from error but minimize its impact significantly. Figure 3 is filtered, and air density corrected WT power curve.

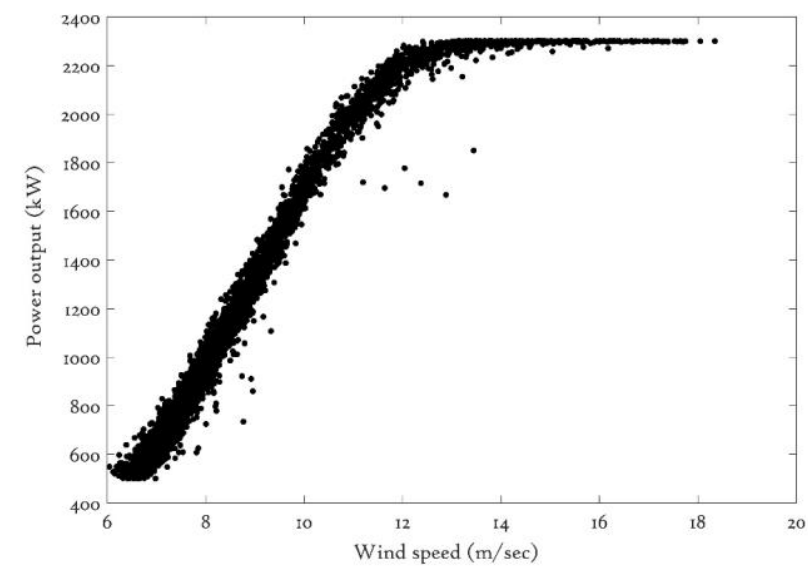


This paper is a post-print of a paper submitted to and accepted for publication in IET Renewable Power Generation and is subject to Institution of Engineering and Technology. To cite the paper please use the doi : 10.1049 /iet-rpg.2018.5728 provided on the IET Digital Library page.

Figure 3: Preprocessed and air density corrected power curve

To analyze the performance of WTs, the SCADA data of 2.3 MW Siemens collected at a wind farm located in Scotland, UK has been used. SCADA contains 13,250 data point that beginning with time stamp " $1 / 10 / 2012$ 00:00 AM" and ending at time stamp " $31 / 12 / 2012$ 23:50 PM" and is sampled at 10-minute average. These measured data points became 3960 data points after pre-processing (Table 1) and will be used for power curve modeling based on advanced similarity between two points. The mean function $\mu(\mathrm{x})$ for the simplicity often taken as zero because preferable to center observed output to have a zero mean although it can be arbitrarily selected. The covariance function is the heart of GP model and generally divided into stationary and nonstationary functions and are explained briefly in [34]. The most widely used covariance function is squared exponential function is a stationary function and would be used in this paper as it is more suitable in expressing the nonlinear relationship of wind speed and power output of a wind turbine and is mathematically expressed as,

Table 1 SCADA data description

\begin{tabular}{cccccc}
\hline Start timestamp & End timestamp & $\begin{array}{c}\text { Measured } \\
\text { data }\end{array}$ & Filtered data & Training data & Validation data \\
\hline $\mathbf{1 0} / \mathbf{2 0 1 2}$ 00:00 AM & $31 / 12 / 201223: 50 \mathrm{PM}$ & 13250 & 3960 & 2500 & 1460
\end{tabular}

nonparametric approaches in upcoming sections. In total, 13,250 SCADA data points were collected from $1^{\text {st }}$ October 2012 to $31^{\text {st }}$ December 2012 which are divided using 10 - fold cross validation into training and validation datasets with a ratio of $70 \%$ and $30 \%$. The training datasets would be used to train the models while validation datasets would be used to assess the performance of the models.

\section{Wind Turbine Power Curve modelling}

In this section, the algorithmic procedure of the proposed advanced nonparametric power curve modelling approaches is explained in detail. The three advanced nonparametric models, namely; Gaussian Process (GP), Support vector machine (SVM), and Random Forest (RF) were used to construct the power curve of a WT. Out of these three approach, GP and SVM are powerful kernel-based methods while RF technique is based on a regression tree.

\subsection{Power Curve model based on GP}

Gaussian Process (GP) model is a nonparametric, nonlinear approach for accurate function approximation in high-dimensional space. GP models are very flexible, and its brief explanation can be found in [34]. Here, a brief description of GP for WTs power curve provided. GP models entirely specified by its mean function and covariance functions,

$$
\mathrm{f}(\mathrm{x}) \sim \mathrm{GP}\left(\mu(\mathrm{x}), \mathrm{k}\left(\mathrm{x}, \mathrm{x}^{\prime}\right)\right)
$$

where the mean function $\mu(\mathrm{x})$ and the covariance function $\mathrm{k}\left(\mathrm{x}, \mathrm{x}^{\prime}\right)$ are defined by,

$$
\begin{gathered}
\mu(\mathrm{x})=\mathrm{E}|\mathrm{f}(\mathrm{x})| \\
\mathrm{k}\left(\mathrm{x}, \mathrm{x}^{\prime}\right)=\mathrm{E} \mid(\mathrm{f}(\mathrm{x})-\mu(\mathrm{x}))\left(\mathrm{f}\left(\mathrm{x}^{\prime}\right)-\mu\left(\mathrm{x}^{\prime}\right)\right)
\end{gathered}
$$

The GP models accuracy depends on its covariance function or kernels (a positive-definite function) and is describes the

$$
\mathrm{k}_{\mathrm{SE}}\left(\mathrm{x}, \mathrm{x}^{\prime}\right)=\sigma_{\mathrm{f}}^{2} \exp \left(-\frac{\left(\mathrm{x}-\mathrm{x}^{\prime}\right)^{2}}{2 \mathrm{1}^{2}}\right)
$$

The squared exponential covariance function $\left(\mathrm{k}_{\mathrm{SE}}\right)$ is a stationary covariance that calculates the covariance between any two points and is a function of Euclidean distance. The wind turbines SCADA datasets come with noise and measurement error which eventually will affect the GP model accuracy. Hence it is advisable to add a noise term added along with covariance function to minimize the impact of these errors and equation (7) further modified to,

$\mathrm{k}_{\mathrm{SE}}\left(\mathrm{x}, \mathrm{x}^{\prime}\right)=\sigma_{\mathrm{f}}^{2} \exp \left(-\frac{\left(\mathrm{x}-\mathrm{x}^{\prime}\right)^{2}}{2 \mathrm{I}^{2}}\right)+\sigma_{\mathrm{n}}^{2} \delta\left(\mathrm{x}, \mathrm{x}^{\prime}\right)$

where $\sigma_{\mathrm{f}}^{2}$ and $\mathrm{l}$ are defined as the hyper-parameters. $\sigma_{\mathrm{f}}^{2}$ describe the signal variance and $l$ is a characteristic length scale which signifies how quickly the covariance decreases with the distance between points.

To estimate the power curve of a WT for given SCADA datasets involves finding the most appropriate parameters and it is generally achieved by maximum log marginal likelihood or posterior estimation. Let us assume that we have a training set $\mathcal{D}$ of $\mathrm{n}$ observations, $\mathcal{D}=$ $\left\{x_{i}, y_{i}\right\}_{i=1}^{n}$. where $x$ an input vector of $D$ and $y$ is a scalar output. The whole input datasets is represented by a $\mathcal{D} \times \mathrm{n}$ matrix since we have $n$ cases of $x$ and with target values collected in a vector $\mathrm{y}$, can be written as $\mathcal{D}=(\mathrm{X}, \mathrm{y})$. Therefore, the function $\mathrm{f}\left(\mathrm{x}_{\mathrm{i}}\right)$ used to transform the input vector $x_{i}$ to the targeted value $y_{i}$ using,

$$
y_{i}=f\left(x_{i}\right)+\epsilon_{i}
$$

where $\epsilon_{\mathrm{i}}$ is Gaussian noise with zero mean and variance $\sigma_{\mathrm{n}}^{2}$ such that $\epsilon_{\mathrm{i}}=\mathrm{N}\left(0, \sigma_{\mathrm{n}}^{2}\right)$. The targeted value $\mathrm{y}$ is a linear combination of Gaussian variables and hence is itself Gaussian [35]. To gather the information about an uncertain parameter, prior distribution used which can be either uninformative or informative. The obtain prior with probability distributions of new data points used to calculate the posterior distribution. The prior on y becomes: 
This paper is a post-print of a paper submitted to and accepted for publication in IET Renewable Power Generation and is subject to Institution of Engineering and Technology. To cite the paper please use the doi : 10.1049 /iet-rpg.2018.5728 provided on the IET Digital Library page.

$$
\begin{gathered}
\mathrm{E}|\mathrm{y}|=\mathrm{E}|\mathrm{f}+\epsilon|=0 \\
\operatorname{cov}|\mathrm{y}|=\mathrm{K}|\mathrm{X} . \mathrm{X}|+\sigma_{\mathrm{n}}^{2} \mathrm{I}
\end{gathered}
$$

The given training dataset $(\mathrm{X}, \mathrm{x})$ used to train the GP model in order to make an estimation of the targeted variable $f$, for given new input $\mathrm{X}_{*}$. The distribution of with new input can be mathematically expressed as:

$$
\left[\begin{array}{l}
\mathrm{y} \\
\mathrm{f}_{*}
\end{array}\right] \sim \mathrm{N}\left(0,\left[\begin{array}{cc}
\mathrm{K}(\mathrm{X}, \mathrm{X})+\sigma_{\mathrm{n}}^{2} \mathrm{I} & \mathrm{k}\left(\mathrm{X}, \mathrm{x}_{*}\right) \\
\mathrm{k}\left(\mathrm{x}_{*}, \mathrm{X}\right) & \mathrm{k}\left(\mathrm{x}_{*}, \mathrm{x}_{*}\right)
\end{array}\right]\right)
$$

$\mathrm{k}\left(\mathrm{X}, \mathrm{x}_{*}\right)=\left[\mathrm{k}\left(\mathrm{x}_{*}, \mathrm{X}_{1}\right) \mathrm{k}\left(\mathrm{x}_{*}, \mathrm{X}_{2}\right) \mathrm{k}\left(\mathrm{x}_{*}, \mathrm{X}_{3}\right) \ldots \ldots \ldots \mathrm{k}\left(\mathrm{x}_{*}, \mathrm{X}_{\mathrm{n}}\right)\right]^{\mathrm{T}}$ is the covariance's between test and training data points in the form of column vector and denoted by $\mathrm{k}_{* *}$ and, $\mathrm{k}\left(\mathrm{x}_{*}, \mathrm{x}_{*}\right)$ is the auto covariance function of the testing data points. It should be noted that the noise variance $\sigma_{\mathrm{n}}^{2}$ has been added to the diagonal of covariance of $\mu$ in order to get the covariance for $y$. The estimated output as per joint Gaussian distribution is given by,

$$
\begin{gathered}
\overline{\mathrm{f}_{*}}=\mathrm{k}_{*}^{\mathrm{T}}\left(\mathrm{K}+\sigma_{\mathrm{n}}^{2} \mathrm{I}\right)^{-1} \mathrm{y} \\
\operatorname{Var}\left(f_{*}\right)=k\left(x_{*}, x_{*}\right)-k_{*}^{T}\left(K+\sigma_{n}^{2} I\right)^{-1} k_{* *}
\end{gathered}
$$

The obtained mean $\overline{\mathrm{f}_{*}}$ and variance $\operatorname{Var}\left(\mathrm{f}_{*}\right)$ are the estimated values and its associated variance respectively. It should be noted that the calculated mean $\overline{\mathrm{f}}_{*}$ is the continuous merger of the output $y$ while posterior variance $\operatorname{Var}\left(\mathrm{f}_{*}\right)$ is a function of $\mathrm{k}_{*}$, hence it will be inversely proportional to the distance between test and training data points. Using the SCADA data of Table 1 power curve is estimated (in MATLAB) using the equations (13) and (14) and is shown in Figure 4. The figure 4 suggest that GP power curve is smooth and continuous and, able to estimate measured power curve accurately.

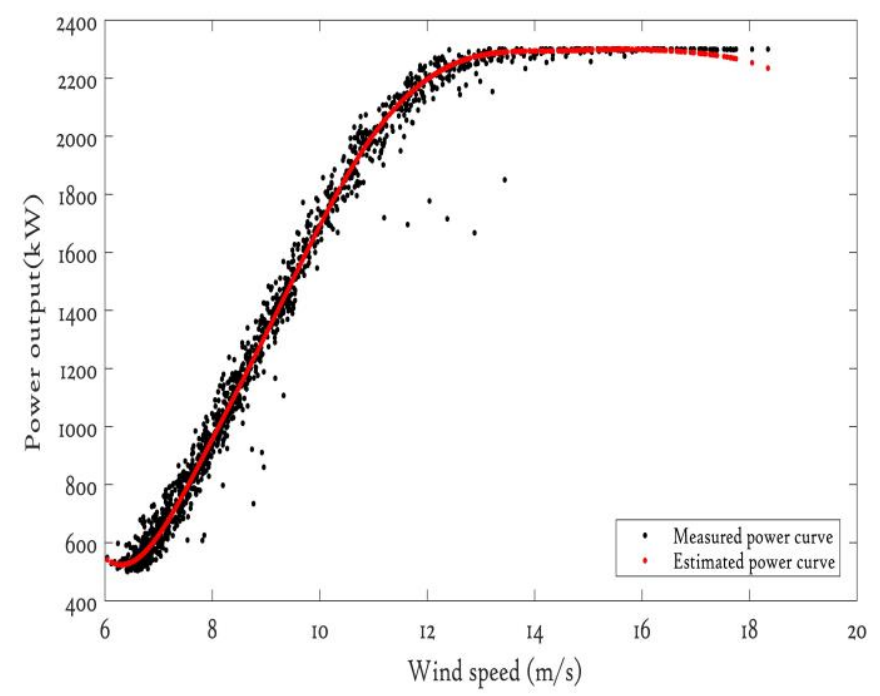

Figure 4: Gaussian Process power curve

It is worth noting, the posterior computation in Gaussian Process regression is a trivial matter but suffers from two issues. The first is that computation mean and variance of equation (13) and (14) requires a matrix inversion and thus has asymptotic complexity called cubic inversion $\mathrm{O}(\mathrm{n})^{3}$ where $n$ is the number of data points. The computational cost increases rapidly with $n$ that it becomes challenging to fit GP models. The last issue is that if $n$ becomes large, then the computation of the $\mathrm{n} \times \mathrm{n}$ matrix becomes problematic and leads to GP model inaccuracy. These two limitation makes GP less attractive. However, techniques based on state-space $[36,37]$ proposed to solve this issue but these methods require high processing power. Hence striking the balance between the data points is the key for constructing an effective GP algorithm for the purpose of fault detection.

\subsection{Power Curve model based on SVM}

The Support vector machine (SVM) is a nonparametric, machine learning technique which follows the principle of structural risk minimization and widely used in solving a problem related to classification (called support vector classification) and regression (called support vector regression). SVM based on statistical learning theory and Ref. $[38,39]$ provide a detailed explanation of SVM. A so-called dual support vector regression (SVR) algorithm used where the inner product of predictors replaced by its corresponding element from the Gram matrix to build a power curve model. The Gram matrix is an n-by-n matrix which contains elements; $g_{i j}=G\left(x_{i}, x_{j}\right)$, where $x_{i}, x_{j}$ are the training SCADA data points. Using nonnegative multipliers, the lagrangian function of the primal function constructed. This Lagrange dual formulation complements the nonlinear system and hence taken into this study. A nonlinear SVR calculates the optimal function $f(x)$ in the transformed predictor space where the SVR search for the coefficient that minimizes the Lagrangian function using the dual formula [38],

$$
\begin{aligned}
& \mathrm{L}(\alpha)=0.5 \sum_{\mathrm{i}=1}^{\mathrm{N}} \sum_{\mathrm{j}=1}^{\mathrm{N}}\left(\alpha_{\mathrm{i}}-\alpha_{\mathrm{i}}^{*}\right)\left(\alpha_{\mathrm{j}}-\alpha_{\mathrm{j}}^{*}\right) \mathrm{G}\left(\mathrm{x}_{\mathrm{i}}, \mathrm{x}_{\mathrm{j}}\right)+ \\
& \varepsilon \sum_{\mathrm{i}=1}^{\mathrm{N}}\left(\alpha_{\mathrm{i}}+\alpha_{\mathrm{i}}^{*}\right)-\sum_{\mathrm{i}=1}^{\mathrm{N}} \mathrm{y}_{\mathrm{i}}\left(\alpha_{\mathrm{i}}-\alpha_{\mathrm{i}}^{*}\right)
\end{aligned}
$$

Under the following constraints:

$$
\begin{aligned}
& \sum_{n=1}^{N}\left(\alpha_{n}-\alpha_{n}^{*}\right)=0 ; \\
& \forall n: 0 \leq \alpha_{n} \leq C ; \\
& \forall \mathrm{n}: 0 \leq \alpha_{\mathrm{n}}^{*} \leq \mathrm{C}
\end{aligned}
$$

The function $f(x)$ used to construct the SVR model for WT power curve and mathematically written as,

$$
f(x)=\sum_{n=1}^{N}\left(\alpha_{n}-\alpha_{n}^{*}\right) G\left(x_{n}, x\right)+b
$$

This specific SVR called $\varepsilon$ - SVR due to its scarcity representation capability [40]. The $\varepsilon$-insensitive loss function is used to build the objective function of the $\varepsilon$ - SVR.

The Karush-Kuhn-Tucker (KKT) conditions play an important role in dealing with constrained optimization and using KKT conditions, [38] of the quadratic programming in which only a certain number of the coefficients $\left(\alpha_{n}-\alpha_{n}^{*}\right)$ will assume nonzero values. The datasets with nonzero coefficients, having approximation errors equal to or larger 
This paper is a post-print of a paper submitted to and accepted for publication in IET Renewable Power Generation and is subject to Institution of Engineering and Technology. To cite the paper please use the doi : $\underline{10.1049 / i e t-r p g .2018 .5728}$ provided on the IET Digital Library page.

than $\varepsilon$, are referred to as support vectors. Other samples are deemed to be $\varepsilon$-insensitive are not support vectors and play no role in the model. Generally, the larger $\varepsilon$, the fewer the number of support vectors and the sparser the representation of the solutions. The KKT complementarity conditions are optimization constraints required to obtain optimal solutions and for nonlinear SVM regression these conditions are:

$$
\begin{aligned}
& \forall \mathrm{n}: \alpha_{\mathrm{n}}\left(\varepsilon+\xi_{\mathrm{n}}-\mathrm{y}_{\mathrm{n}}+\mathrm{f}\left(\mathrm{x}_{\mathrm{n}}\right)\right)=0 ; \\
& \forall \mathrm{n}: \alpha_{\mathrm{n}}\left(\varepsilon+\xi_{\mathrm{n}}^{*}+\mathrm{y}_{\mathrm{n}}-\mathrm{f}\left(\mathrm{x}_{\mathrm{n}}\right)\right)=0 ; \\
& \forall \mathrm{n}: \xi_{\mathrm{n}}\left(\mathrm{C}-\alpha_{\mathrm{n}}\right)=0 ; \\
& \forall \mathrm{n}: \xi_{\mathrm{n}}^{*}\left(\mathrm{C}-\alpha_{\mathrm{n}}^{*}\right)=0
\end{aligned}
$$

The trade-off between the model complexity (flatness) and the degree to which larger deviations tolerated in the optimization formulation is indicated by, [38], whereas controls the width of the $\varepsilon$-insensitive zone and affects the number of support vectors.

The Gaussian kernel is also popularly known by radial basis function (RBF) kernel because it makes computation faster and involves computations in higher dimensional space. In this research, the Gaussian kernel is used model SVR based power curve and mathematically expressed as,

$$
k\left(x, x^{\prime}\right)=\exp \left(-\gamma\left\|x-x^{\prime}\right\|^{2}\right)
$$

where $\gamma$ is the kernel scale for given points $x$ and $x^{\prime}$.

The training and validation SCADA dataset of Table 1 were used to train and test the SVR power curve model, and the result is shown in Figure 5. The SVR based power curve is continuous and accurately predicts the measured power curve. However, at above-rated wind speed, the SVR power curve accuracy deteriorates because of the limited number of data points in that wind speed region. This is because the accuracy of the SVR model depends critically upon the quantity and quality of the data. Furthermore, the SVR model suffers from the cubic inversion issue like GP models (see section 5.1). In short, the main drawback was in the lack of control of the number of data points used by the SVR algorithm, leading to a heavy computational load in the case of a large number of training data points.

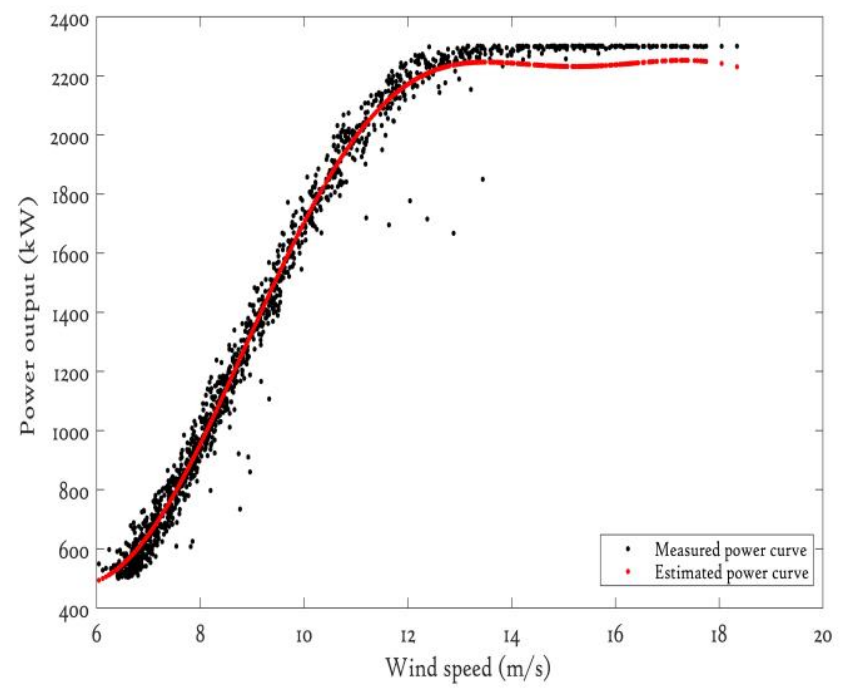

Figure 5: Support Vector Regression based power curve

\subsection{Power Curve model based on RF}

The Random Forest (RF) is a nonparametric, ensemble learning approach that uses a large number of individual, unpruned decision trees and merges together to get a more accurate and stable estimation. The systematic and detailed explanation of RF can be found in $[23,30]$. Here, a brief description of RF would be provided. The RF is a collection of collection of Classification and Regression Trees (CARTs) in which CART splits the input space recursively, according to a predefined split criterion, to small rectangular regions and then fits a simple model, commonly a constant value, in each one of them, and this can be demonstrated by tree diagram, see Figure 6.

In RF ensemble learning approach, a group of 'weak learners' used together to form a 'strong learner' to improve the performance. RF uses decision tree in which each tree is constructed from a bootstrap sample from the original dataset with an objective to increase diversity between members of the ensemble by restricting classifiers to work on different random subsets of the full feature space [42]. In the RF approach, $k$ bootstrap sampled randomly and then a regression tree fit on each sample. After that, average values of $k$ regression tree are taken in order to make an estimation. 
This paper is a post-print of a paper submitted to and accepted for publication in IET Renewable Power Generation and is subject to Institution of Engineering and Technology. To cite the paper please use the doi : $\underline{10.1049 / i e t-r p g .2018 .5728}$ provided on the IET

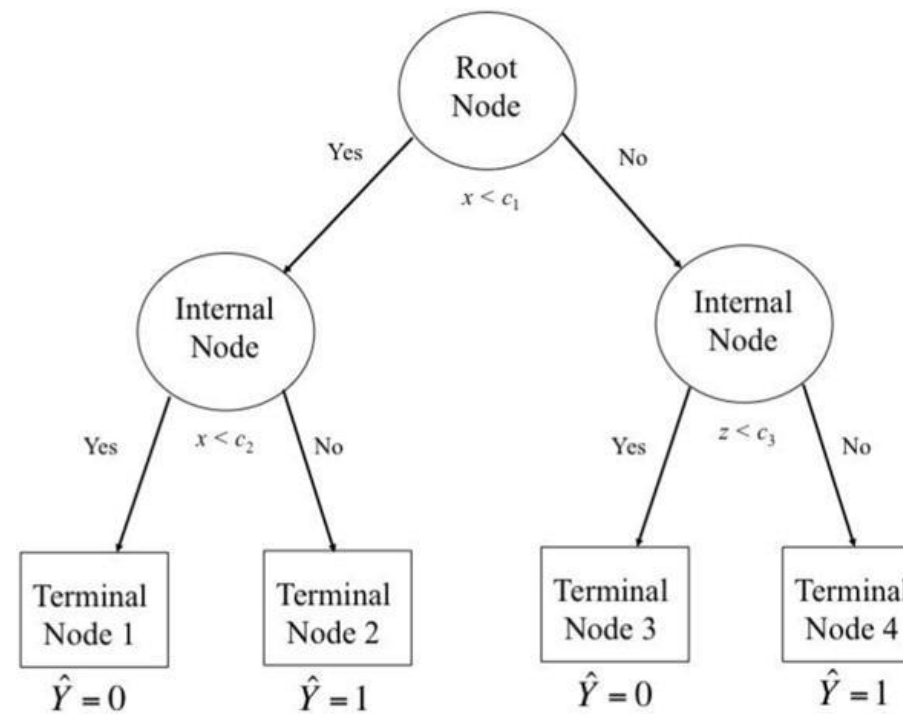

Figure 6: Example tree diagram from classification and regression tree (CART) analysis, [41]

In this paper, RF algorithm as per [43], used to estimate power curve of a WT where bootstrap samples are generated similar to bagging algorithm. The bootstrap aggregation produces non-correlated trees through different training samples which gives immunity to noise. But, instead of using all training data to fit the tree, only random predictor variables are used at each split. Splitting the decision improve the RF accuracy such that the reduction in the residual sum of squares is maximized [44]. Here, SCADA datasets divided into a training and validation datasets (Table 1) at the first node, all variables and values are considered and after the split, further variable and splitting condition is selected and this repeated again. While doing this, same variable can be selected consecutively and hence this splitting technique called recursive binary splitting. To find the optimal values, randomly selected predictor $\left(k_{t r}\right)$ can vary. It should be noted that RF tried to search for the best split among the $k_{t r}$ selected features and this selection is uniform. The randomly selected predictor $\left(k_{t r}\right)$ is same for all prediction trees and it is recommended to be the square root or one third of the features number $k$ as: $k_{t r}=\sqrt{k}$ or, $k_{t r}=\frac{k}{3}$. After that RF algorithm is similar to the CART where by minimizing the cost function, best split is obtained and repeat the procedure until full development of all trees. RF models are very good at capturing the nonlinear relationship between features and the target and in minimizing the overfitting issue. The out-ofbag error (OOBE) and the measure of variable importance (VI) are the two main important properties of RF models, see [23]. The spatial 10-minute average training SCADA datasets (Table 1) are used to estimate the power curve based on RF and is shown in Figure 7. Figure 7 suggest that the RF based power curve is accurately following measured power curve variance but neither it is continuous or smooth. It is worth to note that, the RF power curve is predictive model, not a descriptive model and hence it does not give description of the relationship among the predictors. Moreover, confusing data makes RF inaccurate and confusing, hence it is desirable to select appropriate and error free predictors that affect the target variable.

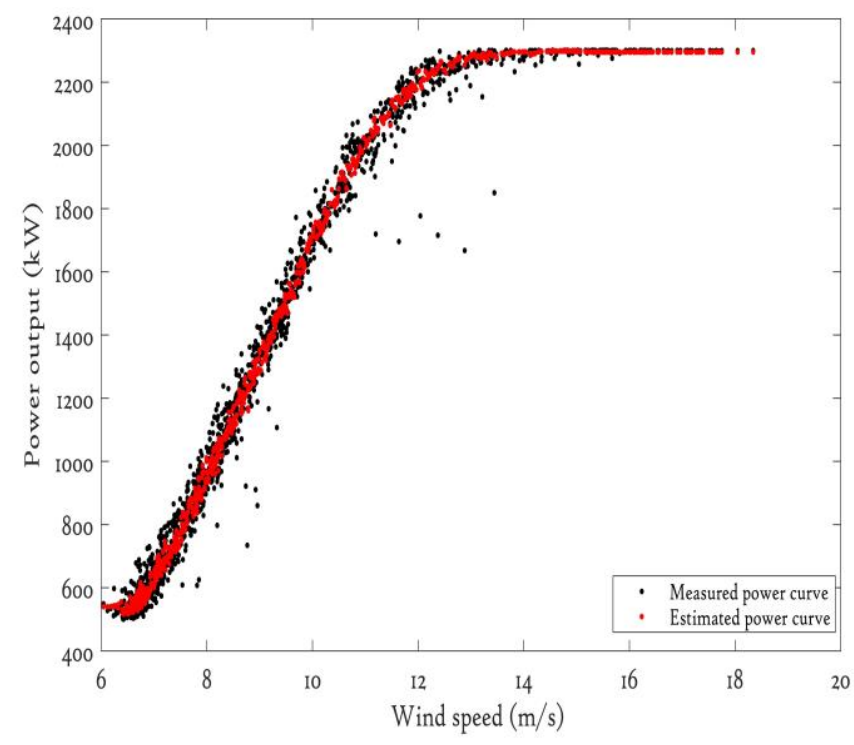

Figure 7: Random Forest based power curve

\section{Performance comparison}

The advanced nonparametric models discussed in Section 5 are used for comparative analysis in order to find out which of the proposed advanced nonparametric models is appropriate to represent power curves accurately and what are advantageous and disadvantageous of proposed advanced nonparametric models. The residual analysis, performance error metrics are used for comparative studies of proposed models. The advanced nonparametric models result presented in Section 5 were compared in Figure 8 together with the measured power curve. The GP based power curve is relatively more accurate and have continuous and smooth fitting which closely following the expected variance at all wind speed range while RF based power curve is neither continuous nor smooth because it is built on CART theory $[23,30]$ but closely matching the measured power curve. The performance of power curve based on SVM deteriorates after rated wind speed because of the unavailability of reasonable numbers of SCADA data points as shown in Figure 8. Figure 9 shows the estimated power values in the time series of proposed advanced nonparametric models. 
This paper is a post-print of a paper submitted to and accepted for publication in IET Renewable Power Generation and is subject to Institution of Engineering and Technology. To cite the paper please use the doi : $\underline{10.1049 / i e t-r p g .2018 .5728}$ provided on the IET

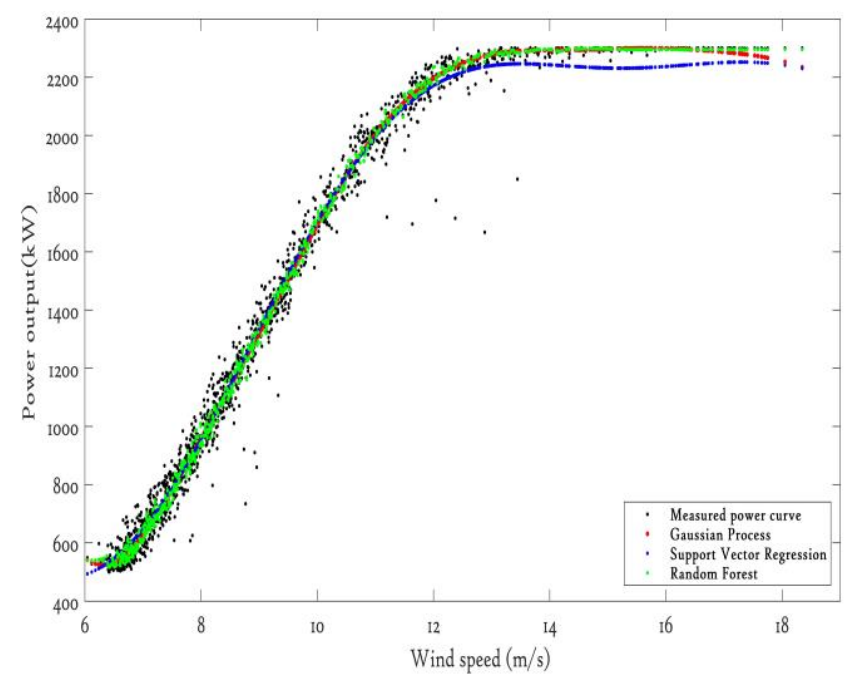

Figure 8: Comparative analysis of nonparametric models

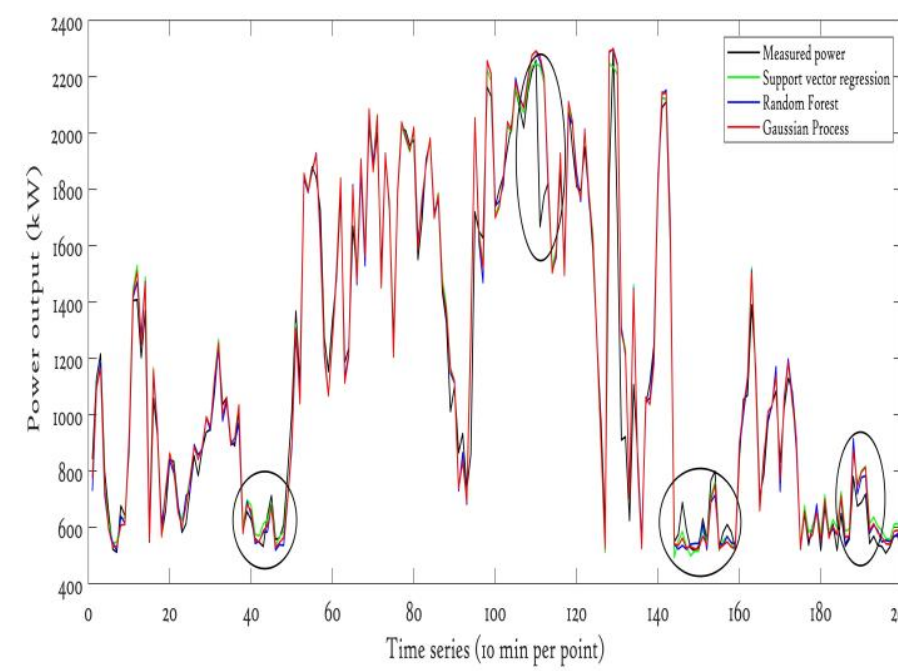

Figure 9: Comparative analysis of nonparametric models in terms of time series

\subsection{Using Performance error metrics}

The Random Forest (RF) There are several statistical performance metrics that can be used to measure performance of the estimated power curves such as the root-mean-squared error (RMSE), normalized mean absolute percentage error (NMAPE), symmetric mean absolute percentage error (sMAPE), the mean absolute error (MAE), and the coefficient of determination (R2), [47]. In this paper, we use three goodness-of-fit indicators, namely the mean absolute error (MAE), the root mean squared error (RMSE), and coefficient of determination $\left(R^{2}\right)$ to evaluate the goodness-of-fit statistics of the advanced nonparametric power curve models which are mathematically expressed as,

$$
\begin{aligned}
\text { MAE } & =\frac{\sum_{\mathrm{i}=1}^{\mathrm{n}} \mathrm{abs}\left(\mathrm{X}_{\mathrm{i}}{ }^{\prime}-\mathrm{X}_{\mathrm{i}}\right)}{\mathrm{n}} \\
\text { RMSE } & =\sqrt{\frac{\sum_{\mathrm{i}=1}^{\mathrm{n}}\left(\mathrm{X}_{\mathrm{i}}^{\prime}-\mathrm{X}_{\mathrm{i}}\right)^{2}}{\mathrm{n}}} \\
\mathrm{R}^{2} & =1-\frac{\mathrm{SSE}}{\mathrm{TSS}}
\end{aligned}
$$

where $X^{\prime}$ are the predicated values for $n$ different predictions, and $X$ are the measured values. SSE is the sum of squared errors and TSS, is the total sum of squares.

The RMSE is the square root of the mean of the squared difference between the measured and predicted values of power. The MAE is the mean of the absolute values of the differences between the measured and predicted values of power. The coefficient of determination $\left(R^{2}\right)$, describes how close the data are to the fitted nonparametric models and is calculated as the square of the correlation between estimated output and measured values using equation (21). The RMSE, MAE and $R^{2}$ values of the discussed algorithms have been tabulated in table 2 (see appendix). The lower values of RMSE or MAE suggest better estimation of power curve while a higher value of $\mathrm{R}^{2}$ indicates a better coincidence of observed and estimated results. Based on these three performance metrics, GP algorithm rank 1 and gives the most accurate power curve while RF based power curve ranks 3 and relatively gives inaccurate power curve.

\subsection{Using models Residuals Analysis}

The GP, RF, and SVM models are data-driven, nonlinear techniques whose residual distribution needs to be analyzed. Residuals is the difference between measured value and estimated values and can be useful in identifying the deviation between the data and the regression model which widely used to measure the variability in the response variable. The frequency distribution of the calculated residuals of advanced nonparametric models is shown in Figure 10 together with a fitted Gaussian distribution and found that distribution of GP residuals is close to Gaussian as compare to other nonparametric models.
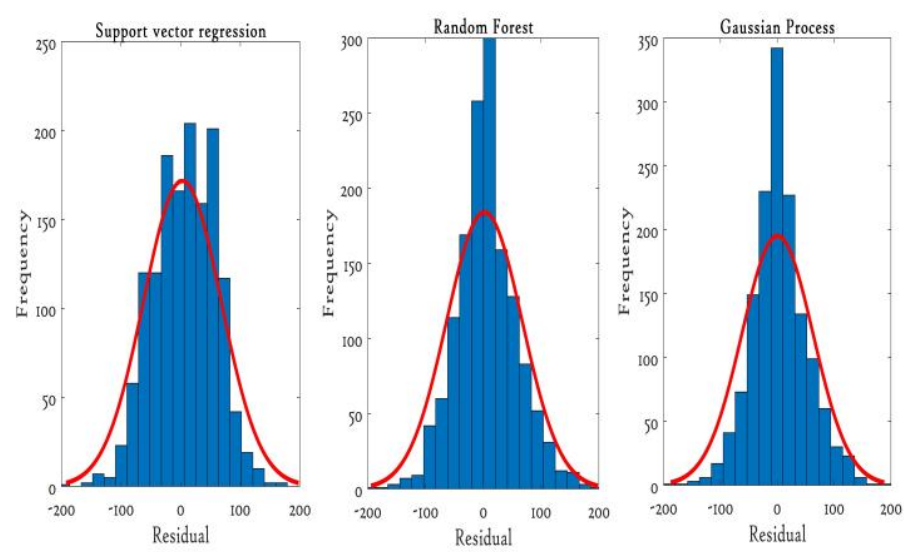

Figure 10: Comparative studies of histogram fitting of nonparametric models

\subsection{Using models Uncertainty}

The Random Forest Wind turbine power curve vastly used by wind industries to identify the failures that cause the turbine to underperform and do preventive maintenance in order to prevent downtime and catastrophic stage. Uncertainty analysis is significant for constructing robust fault detection algorithm based on WT power curve. The GP estimate confidence intervals (CIs) along with the mean function which makes uncertainty analysis is simple and straightforward. The GP CIs is calculated by the standard 
This paper is a post-print of a paper submitted to and accepted for publication in IET Renewable Power Generation and is subject to Institution of Engineering and Technology. To cite the paper please use the doi : 10.1049 /iet-rpg.2018.5728 provided on the IET Digital Library page.

deviation of the variance of the estimated function (equation 14) using equation (18),

$$
\text { CIs }=\overline{\mathrm{f}_{*}} \pm 2 \sqrt{\operatorname{Var}\left[\mathrm{f}_{*}\right]}
$$

The equation (18) conclude that GP CIs is the pointwise mean plus and minus two times the standard deviation for given input data points and it corresponds to $95 \%$ confidence region which defines the significance level of 0.05 for the calculated prior and posterior respectively. The \pm of the CIs used to represent the upper and lower confidence interval of the GP model respectively. The data points lie outside these CIs likely to suggest turbine underperformance and hence vital for fault detection algorithms based on the GP model. However, in the case of RF and SVM models, uncertainty analysis is complicated due to the extra mathematical challenges associated with it. Some authors proposed techniques to calculate the confidence intervals for Random Forest [45] and SVM [46] models but that requires high power processing, and computational cost and consequently makes the O\&M cost higher.

\section{Conclusions}

The performance of WT can be described by power curve, and therefore, accurate modeling of the power curve is essential for assessment and monitoring of the turbine's performance, energy warranty formulations, power forecasting, as well as sizing the storage capacity for wind power integration. The advanced nonparametric models (GP, SVM, and RF) for estimating WT power curves based on SCADA datasets obtained from operational WTs are presented in this paper. GP and SVM are kernel methods while RF is a regression tree method inspired by CART principle. The Computational results have demonstrated that the GP has highest fitting accuracy, and able to reflect the dynamic properties of a power curve whose distribution function is close to the Gaussian distribution. Furthermore, the accuracy of each method is evaluated by the performance error metrics (RMSE, MAE and $R^{2}$ ) and based on that GP model ranks 1(see Table 2). The accuracy of SVM model suffers at above rated wind speed because of unavailability of a reasonable number of SCADA data points while with same SCADA data points, GP perform better across all wind speed range (including above rated wind speed). The uncertainty analysis in GP model is done by confidence intervals which is simple and straightforward which makes fault detection GP algorithm robust and free from further complex mathematical calculations unlike RF and SVM methods where the uncertainty analysis leads to extra mathematical computations and cost. This makes both RF and SVM are less attractive for WT condition monitoring activities from economic as well as technical point of view.

The future work is to use these proposed model to construct WT fault detection algorithms and find out which model is effective in identifying failures without any false positives.

\section{Acknowledgments}

This project has received funding from the European Union's Horizon 2020 research and innovation programme under the Marie Sklodowska-Curie grant agreement No 642108.

\section{References}

[1] Bently, D., and Hatch, C.: Fundamentals of Rotating Machinery Diagnostics. ASME publishers, New York, 2003. [2] Qiao,W., Lu, D.: 'A survey on wind turbine condition monitoring and fault diagnosis- Part II: signals and signal processing methods'. IEEE Transactions on Industrial Electronics, 2015, 62, (10), pp 6546-6557.

[3] Márquez, F., Tobias, A., Pérez, J., Papaelias, M., : 'Condition monitoring of wind turbines: techniques and methods'. Renewable Energy, 2012, 46, pp 169-178.

doi: 10.1016/j.renene.2012.03.003.

[4] Dai, J., Yang, W., Cao,J., Liu,D., Long,X., : 'Long Ageing assessment of a wind turbine over time by interpreting wind farm SCADA data'. Renewable Energy, 2018,116 (Part B), pp. 199-208. doi :10.1016/j.renene.2017.03.097.

[5] Dao, P., Staszewski, W., Barszcz, T., Uhl, T., 'Condition monitoring and fault detection in wind turbines based on cointegration analysis of SCADA data'. Renewable Energy,2018,16 (Part $\mathrm{B}$ ), pp 107-122. doi: 10.1016/j.renene.2017.06.089.

[6] Yang, W., Lang, Z., Tian, W., 'Condition monitoring and damage location of wind turbine blades by frequency response transmissibility analysis. IEEE Transactions on Industrial Electronics,2015, 62, (10), pp 6558-6564. doi: 10.1109/TIE.2015.2418738.

[7] Tang, J., Soua, S., Mares, C., Gan, Tat-Hean.,: 'An experimental study of acoustic emission methodology for in service condition monitoring of wind turbine blades'. Renewable Energy, 2016,99, pp 170-179. doi: doi.org/10.1016/j.renene.2016.06.048.

[8] Ifigeneia, A.: 'Accounting for Nonstationarity in the Condition Monitoring of Wind Turbine Gearboxes'. Ph.D. thesis, University of Sheffield, 2013.

[9] Lei,Y., Lin,J., He,Z., Zuo,M., : 'A review on empirical mode decomposition in fault diagnosis of rotating machinery'. Mechanical Systems and Signal Processing. 2013,35, (1), pp:108-126. doi: 10.1016/j.ymssp.2012.09.015.

[10] Yang, D., Li, H., Hu, Y., Zhao, J., Xiao, H., Lan, Y.,: ' Vibration condition monitoring system for wind turbine bearings based on noise suppression with multipoint data fusion'. Renewable Energy, 2016, 92, pp 104-116. doi: doi.org/10.1016/j.renene.2016.01.099.

[11] Castellani, F., Astolfi, D., Sdringola, P., Proietti, S., Terzi, L.,: 'Analyzing wind turbine directional behavior: SCADA data mining techniques for efficiency and power assessment'. Applied Energy, 2015,185(Part 2), pp 10761086. doi: doi.org/10.1016/j.apenergy.2015.12.049.

[12] Khalfallah, M., Koliub, A.,: 'Suggestions for improving wind turbines power curves'. Desalination, 2007, 209, (3), pp. 221-229, doi: doi.org/10.1016/j.desal.2007.04.031.

[13] Giorsetto, P., Utsurogi, K.,: 'Development of a new procedure for reliability modeling of wind turbine generators'. IEEE Transactions on Power Apparatus and Systems, 1983, PAS-102, (1), pp. 134-143, doi: 10.1109/TPAS.1983.318006. [14] R. Chedid, H. Akiki, and S. Rahman, "A decision support technique for the design of hybrid solar-wind power 
This paper is a post-print of a paper submitted to and accepted for publication in IET Renewable Power Generation and is subject to Institution of Engineering and Technology. To cite the paper please use the doi : 10.1049 /iet-rpg.2018.5728 provided on the IET Digital Library page.

systems," IEEE Trans. Energy Convers., vol. 13, no. 1, pp. 76-83, Mar. 1998.

[15] Kusiak, A., Zheng, h., Song, Z.,:'On-line monitoring of power curves'. Renewable Energy, 2009,34, (6), pp. 14871493, doi: doi.org/10.1016/j.renene.2008.10.022.

[16] Lydia, M., Kumar, S., Selvakumar,A., Kumar, A.,: ‘ A comprehensive review on wind turbine power curve modeling techniques'. Renew Sustain Energy Review, 2014, 30, pp. 452-460.doi: doi.org/10.1016/j.rser.2013.10.030.

[17] Gill, S., Stephen, B., Galloway, S.,: 'Wind Turbine Condition Assessment Through Power Curve Copula Modeling'. IEEE Transactions on Sustainable Energy, 2012,3, (1), pp. 94-101, doi: 10.1109/TSTE.2011.2167164.

[18] Shokrzadeh, S., Jozani, M., Bibeau, E.,: 'Wind Turbine Power Curve Modeling Using Advanced Parametric and Nonparametric Methods'. IEEE Transactions on Sustainable Energy, 2014, 5, (4), pp. 1262-1269, doi: 10.1109/TSTE.2014.2345059.

[19] Kou, K., Gao, F., Guan, X.,: 'Sparse online warped Gaussian process for wind power probabilistic forecasting'. Applied Energy, 2013, 108, pp. 410-428, doi: doi.org/10.1016/j.apenergy.2013.03.038

[20] Díaz, S., Cartaa, J., Matías. J.,: 'Performance assessment of five MCP models Proposed for the estimation of long-term wind turbine power outputs at a target site using three machine learning techniques'. Applied Energy, 2018, 209, pp. 455-477, doi: doi.org/10.1016/j.apenergy.2017.11.007

[21] Jung, S., Kwon, S.,: 'Weighted error functions in artificial neural networks for improved wind energy potential estimation'. Applied Energy, 2013, 111, pp. 778-790, doi: doi.org/10.1016/j.apenergy.2013.05.060.

[22] Marvuglia, A., Messineo, A.,: 'Monitoring of wind farms' power curves using machine learning techniques'. Applied Energy, 2012, 98, (0), pp. 574-583, doi: doi.org/10.1016/j.apenergy.2012.04.037.

[23] Lahouar, A., Slama, J.,: 'Hour-ahead wind power forecast based on random forests'. Renewable Energy, 2017, 109, pp. 529-541, doi: doi.org/10.1016/j.renene.2017.03.064. [24] Zhang, Y., Wang, J.,: 'K-nearest neighbors and a kernel density estimator for GEFCom 2014 probabilistic wind power forecasting'. International Journal of Forecasting,2016, 32, (3), pp. 1074-1080, doi: doi.org/10.1016/j.ijforecast.2015.11.006.

[25] Meer, D., Widén, J., Munkhammar, J.,: “ Review on probabilistic forecasting of photovoltaic power production and electricity consumption'. Renewable and Sustainable Energy Reviews, 2018,81, (Part 1), pp. 1484-1512, doi: doi.org/10.1016/j.rser.2017.05.212.

[26] Hong, T., Fan, S.,: 'Probabilistic electric load forecasting: a tutorial review'. International Journal of Forecasting, 2016, 32, (3), pp. 914-938, doi: 10.1016/j.ijforecast.2015.11.011.

[27] Cortes, C., Vapnik, V.,: 'Support-vector networks'. Machine learning, 1995, 20, (3), pp. 273-297, doi: doi.org/10.1007/BF00994018.

[28] Baccarini, L., Silva, V., Menezes, B., Caminhas, W.,: 'SVM practical industrial application for mechanical faults diagnostic'. Expert Systems with Applications, 2011, 38, (6), pp. 6980-6984, doi: doi.org/10.1016/j.eswa.2010.12.017.

[29] Lin, Y., Kruger, U., Zhang, J., Wang, Q., Lamont, L., Chaar, L.,: 'Seasonal analysis and prediction of wind energy using random forests and arx model structures. IEEE Transactions on Control Systems Technology, 2015, 23, (5), pp.1994-2002. Doi: 10.1109/TCST.2015.2389031.

[30] Freund, Y.,: 'Boosting a weak learning algorithm by majority'. Information and Computation, 1995, 121, (2), pp. 256-285, doi: doi.org/10.1006/inco.1995.1136.

[31] Paiva, L., Rodrigues, C., Palma, J.: 'Determining wind turbine power curves based on operating conditions'. Wind Energy, 2014, 17, pp. 1563-1575, doi: doi.org/10.1002/we.1651.

[32] IEC 61400-12-1: 'Wind Turbines-Part 12-1: Power Performance Measurements of Electricity Producing Wind Turbines', 2006.

[33] Schlechtingen, M., Santos, I.,: 'Comparative analysis of neural network and regression based condition monitoring approaches for wind turbine fault detection'. Mechanical system signal processing, 2011, 25, (5), pp. 1849-1875, doi: doi.org/10.1016/j.ymssp.2010.12.007.

[34] Rasmussen, C., Williams, C.,:'Gaussian Processes for machine learning'. The MIT Press, 2006, ISBN 026218253X. [35] Chatfield, C., Collins, A.,: 'Introduction to Multivariate Analysis'. Springer, 1980.

[36] Hartikainen, J.,Särkkä, S.,: 'Kalman filtering and smoothing solutions to temporal Gaussian process regression models'. IEEE International Workshop on Machine Learning for Signal Processing, Kittila, 2010, pp. 379-384. doi: 10.1109/MLSP.2010.5589113.

[37] Sarkka, S., Solin, A., Hartikainen,J.,: 'Spatiotemporal learning via infinite-dimensional Bayesian filtering and smoothing'. IEEE Signal Processing Magazine, 30, (4), pp. 51-61, doi: 10.1109/MSP.2013.2246292.

[38] Boser, B. E.; Guyon, I. M.; Vapnik, V.,: 'A training algorithm for optimal margin classifiers'. Proceedings of the fifth annual workshop on Computational learning theory COLT, 1992, pp. 144. ISBN 089791497X. doi:10.1145/130385.130401.

[39] Fan, R.E., Chen, P., Lin. C.,: 'A Study on SMO-Type Decomposition Methods for Support Vector Machines'. IEEE Transactions on Neural Networks, 2006, 17, (4), pp. 893-908, doi: 10.1109/TNN.2006.875973.

[40] Cherkassky, V., Ma, Y.,: 'Practical selection of SVM parameters and noise estimation for SVM regression'. Neural Networks, 2004, 17, (1), pp 113-126, doi: doi.org/10.1016/S0893-6080(03)00169-2.

[41] Berk, R.,: 'Statistical learning from a regression perspective'. New York, Springer; 2009.

[42] Sammut, C., Webb, G.,: 'Encyclopedia of Machine Learning and Data Mining'. Springer, 2017, Boston, MA.

[43] Breiman L. Random forests. Mach Learn 2001;45(1):532.

[44] Breiman, L., Friedman, J., Olshen, R., Stone, C.,: 'Classification and regression trees'. New York: Chapman and Hall,1984.

[45] Wager, S., Hastie, T., Efron, B.,: 'Confidence Intervals for Random Forests: The Jackknife and the Infinitesimal Jackknife'. Journal of Machine Learning Research,2014,15, pp.625-1651. Available online at http://www.jmlr.org/papers/volume15/wager14a/wager14a.p df. (accessed on 20th July 2018). 
This paper is a post-print of a paper submitted to and accepted for publication in IET Renewable Power Generation and is subject to Institution of Engineering and Technology. To cite the paper please use the doi : $\underline{10.1049 / i e t-r p g .2018 .5728}$ provided on the IET Digital Library page.

[46] Jiang,B., Zhang, X., Cai, T.,: 'Estimating the Confidence Interval for Prediction Errors of Support Vector Machine Classifiers'. Journal of Machine Learning Research, 2008, 9, pp. 521-540. Available online at http://www.jmlr.org/papers/volume9/jiang08a/jiang08a.pdf. (accessed on 20th July 2018).

[47] A. Marvuglia and A. Messineo. Monitoring of wind farms power curves using machine learning techniques. Appl.

Energy, vol. 98, no. 0, pp. 574-583, 2012.

\section{APPENDIX}

Table 2 Evolution of nonparametric models using performance metrics

\begin{tabular}{ccccc}
\hline MODELS & RMSE & $\boldsymbol{R}^{\mathbf{2}}$ & MAE & RANK \\
& & & & \\
\hline GP & 62.690 & 0.990 & 39.806 & 1 \\
\hline SVM & 65.086 & & & \\
\hline RF & 65.444 & 0.989 & 46.226 & 3
\end{tabular}

